\title{
Estructura factorial del Mindful Attention and Awareness Scale para estudiantes mexicanos de nivel medio superior ${ }^{1}$
}

\author{
Factorial structure of the Mindful Attention \\ and Awareness Scale for Mexican students
}

\author{
Fabián Israel Balderas Molina ${ }^{2}$, Angélica Riveros Rosas ${ }^{3}$ \\ y Ana Beatriz Moreno Coutiño ${ }^{2}$
}

\begin{abstract}
RESUMEN
El Mindful Attention and Awareness Scale es el instrumento más utilizado en el mundo para evaluar la atención plena. Ha sido adaptado a población adolescente en algunos países, mostrando una estructura unifactorial de atención-reconocimiento. Sin embargo, se ha indicado también la contribución de un segundo factor que aporta una mayor varianza al instrumento. El objetivo de este estudio fue obtener la estructura factorial del citado instrumento para estudiantes de nivel medio superior a través de un análisis factorial confirmatorio. La población de estudio fue de 267 estudiantes de ambos sexos, con una edad de entre 15 y 21 años. El análisis factorial confirmatorio se integró por dos factores: atención y reconocimiento. El índice de consistencia interna del instrumento tuvo un coeficiente alfa de 0.82 y 0.73 , respectivamente. Además, el alfa total fue elevado. Los índices de ajuste fueron superiores para un modelo de dos factores respecto al modelo unifactorial. La version final del instrumento se conformó por doce reactivos.
\end{abstract}

Palabras clave: Atención plena; Estudiantes; Evaluación.

\begin{abstract}
The Mindful Attention and Awareness Scale (MAAS) is one of the most commonly used for measuring mindfulness, and has been adapted for adolescent populations in several countries. The scale has consistently shown a single attention-awareness factor, but there is evidence of a second factor that provides a wider variance to the scale. The aim of the present study was to obtain the factorial structure of the MAAS in junior college students through a confirmatory factor analysis. A total of 267 students of both sexes, aged between 15 and 21 years old participated. Confirmatory factor analysis revealed two factors: attention and awareness, with a high internal consistency alpha index. The fitness index model was higher for a two-factor model than for a one-factor model. The final version of the instrument was conformed by twelve items.
\end{abstract}

Key words: Mindfulness; Students; Evaluation.

\footnotetext{
${ }^{1}$ Agradecimientos: Dra. Nazira Calleja- Facultad de Psicología. Dra. Natalia Arias, Lic. Ethelmar Vázquez Pérez, Lic. Diana Rosalba Cortés Monter, Aída Mariana Orozco Arreola y Gabriela Mendoza-Laboratorio de infantes, Facultad de Psicología. Mtro. Juan Santiago, Residencia de Medicina Conductual, Facultad de Psicología. Gueshe Lobsang- Centro Loseling México. Dr. Fernando González Blancas- Servicio Médico Universitario-Universidad Autónoma Chapingo.

${ }^{2}$ Facultad de Psicología, Universidad Nacional Autónoma de México, Av. Universidad 3004, Col. Copílco Universidad, Del. Coyoacán, 04510 Ciudad de México, México, correo electrónico: israebm@gmail.com. Artículo recibido el 14/agosto de 2017 y aceptado el 5 de enero de 2018. ${ }^{3}$ Facultad de Contaduría y Administración, Universidad Nacional Autónoma de México, Circuito Exterior s/n, Col. Copílco Universidad, Del. Coyoacán, 04510 Ciudad de México, México.
}

Citación: Balderas M., F.I., Riveros R., A. y Moreno C., A.B. (2018). Estructura factorial del Mindful Attention and Awareness Scale para estudiantes mexicanos de nivel medio superior. Psicología y Salud, 28(2), 239-250. 


\section{INTRODUCCIÓN}

$\mathrm{E}$ 1 entrenamiento en mindfulness o atención plena (AP en lo sucesivo) se ha empleado como un conjunto de técnicas cuyo fin es enseñar a las personas a responder de manera adaptativa a diversos estresores emocionales y alteraciones fisiológicas. En los últimos quince años, la investigación sobre la práctica de la AP ha aumentado considerablemente. Por ejemplo, en el periodo comprendido entre los años 2013 y 2016 las bases de datos de EBSCO, PsycInfo y Academic Search Complete indican cerca de 27 mil referencias al utilizar la palabra clave "mindfulness". Es importante señalar que hasta el momento no se tiene una definición aceptada universalmente de "atención plena", puesto que diferentes autores han elaborado su propia traducción y definición del término inglés, lo que dificulta su medición y sus componentes. A pesar de ello, se consideran cuatro elementos de la AP que comparten las distintas definiciones: 1) la conciencia de la propia experiencia, incluidas las sensaciones corporales, pensamientos, emociones y sucesos externos percibidos por los sentidos (p. ej., imágenes, sonidos y aromas); 2) la atención sostenida de estos estímulos; 3) la focalización en el momento presente, y 4) la aceptación de esos estímulos sin juzgarlos como agradables o desagradables (Chambers, Gullone y Allen, 2009; Rapgay y Bystrisky, 2009; Teasdale, Segal y Williams, 1995).

A pesar de la dificultad para lograr una definición que sea ampliamente aceptada, cada una de las definiciones comparte tres términos principales: atención, conciencia y reconocimiento, por lo que la AP se describe en su forma más sencilla como la capacidad para mantener la mente consciente en el momento presente (Kabat-Zinn, Lipworth y Burney, 1985). Según Brown y Ryan (2003), la conciencia (consciousness) comprende dos aspectos: awareness, término que traducido al español significa "darse cuenta", que es el aspecto de fondo, o sea, el "sondeo" que la conciencia realiza para supervisar continuamente los estímulos internos y externos, y attention (atención, en español), que se entiende como focalizar de manera consciente la percepción hacia aquello que se está experimentando (Teasdale et al., 1995). Los autores de esta propuesta manifiestan que, mientras que la atención y la percepción son rasgos relativamente constantes en las personas, la conciencia puede ser la vía para mejorar tales rasgos, es decir, "ser consciente," por lo que dichos componentes actúan como un sistema único, considerado como un solo factor. Una característica central de practicar la AP es el mejoramiento del proceso de focalización, reconocimiento y atención dirigidos a una determinada experiencia. Por ello, Brown y Ryan (2003) sugieren que la AP es tanto un rasgo como un estado que interactúan en la autorregulación y la experiencia emocional; es decir, que los participantes responden de manera diferente en cuanto a ser conscientes y a atender lo que les está sucediendo en el presente (rasgo). Asimismo, refieren que la capacidad de la AP varía en cada individuo ya que puede ser intensificada o entorpecida por otros factores (estados). Adicionalmente, consideran que reconocer la atención-emoción es la habilidad de las personas de estar atentas, e implica reconocer el momento presente y estar conscientes de las experiencias mentales, sin generar un juicio de tipo dual "negativo-positivo," "deseado-no deseado" en quienes las experimentan (Brown y Ryan, 2003).

La aplicación de programas de AP en diversos escenarios, en su mayoría clínicos (Gregoski, Barnes, Tingen, Harshfield y Treiber, 2011; Keng, Smoski y Robins, 2011; Santiago, 2016), con personas clínicamente sanas (Campanella, Crescentini, Urgesi y Fabbro, 2014; Khoury, Sharma, Rush y Fournier, 2015) y con estudiantes universitarios (Soler et al., 2012) ha mostrado resultados significativos, pero algunos estudios no reportan la evaluación de la AP (Santiago, 2016). En la literatura científica se han reportado hasta el momento once escalas para medir los efectos de la práctica de la AP. Actualmente, en México solo se dispone de dos instrumentos validados en población mexicana: el Cuestionario de Cinco Factores de AP (FFMQ, por sus siglas en inglés) para evaluar la población universitaria (Meda-Lara, Herrero, Blanco, Moreno y Palomera, 2015), y el Mindful Attention Awarness Scale (MAAS en lo sucesivo) para la población general (López-Maya et al., 2015).

El cuestionario para evaluar la AP más utilizado en investigación, tanto en estudios teóricos 
como en escenarios clínicos, es justamente el MAAS, el cual puede aplicarse a personas con y sin experiencia en la práctica de la AP. Asimismo, existen adaptaciones del MAAS hechas con adolescentes alemanes (Bruin, Zijlstra y Veirjer-Bergsma, 2011) y con población general en España (Inchausti, Prieto y Delgado, 2014; Soler et al., 2012) y México (López-Maya et al., 2015), en los cuales se reporta un factor de atención-reconocimiento con $14,15,12$ y 15 reactivos. Los estudios mencionados refieren que hay indicios de dos factores. Por ejemplo, Bruin et al. (2011) reportan que, al analizar los componentes principales, sus resultados se integraron por dos factores; si bien la varianza explicada de cada componente es amplia, y que el instrumento consta de un solo factor, con un RMSEA (Root mean square error of approximation) de .0 .35 (90\% IC, de .019 a .050) y CFI (Comparative fit index) de 0.991 . Además, dichos autores mencionan que en estudios previos realizados con esta escala se había sugerido la existencia de dos componentes: uno de atención y otro de aceptación, pero este último factor no aportó significativamente a la varianza de la escala.

En cuanto a Soler et al. (2012), consideran el instrumento como de solo un factor, con una varianza acumulada de $42.8 \%$, en la que se mantienen los quince reactivos, con sus índices de ajuste (CFI $=0.920$, GFI [Goodness of fit index] $=0.884$, AGFI [Adjusted goodness of fit index] $=0.845$, SRMR [Standardized root mean square residual $]=0.053$, RMSEA $=0.71)$. Por su parte, Inchausti et al. (2014) muestran que los datos se desvían ligeramente de la unidimensionalidad del instrumento, ya que en su análisis de rachas la varianza fue suficientemente alta en el pretest, con $40.7 \%$, mientras que en el postest fue de 50.9\%, lo cual indica la contribución de un segundo factor a dicha disparidad; la diferencia entre varianzas explica cómo es que algunos pacientes pueden atender el momento presente, pero no necesariamente aceptarlo o reconocerlo, lo que les genera malestar, contrariamente a los pacientes entrenados en AP, quienes logran la aceptación. En cambio, López-Maya et al. (2015) refieren que sus resultados indican tres factores al utilizar un análisis de componentes principales, los que explican $57.66 \%$ de la varianza, aunque el gráfico de sedi- mentación muestra un factor alejado de los demás elementos, por lo que los autores indican un modelo unifactorial que explica $43.62 \%$ de la varianza, con índices de ajuste RMSEA $=0.078(90 \% \mathrm{CI}$ 0.071-0.086), CFI $=0.913$, resultados similares a los Soler et al. (2012).

Tomando en cuenta el contexto anterior, el objetivo del presente trabajo fue esclarecer las diferencias entre los reportes de estudios que sugieren dos o más factores del MAAS, así como obtener las propiedades psicométricas del instrumento para su uso específico en población de nivel educativo medio superior.

\section{MÉTODO}

El estudio consistió en cuatro etapas: 1) validación de jueces expertos (cf. Escobar y Cuervo, 2008), 2) validación del contenido por lingüistas, 3) revisión por jueces expertos y 4) aplicación de la escala revisada a estudiantes de nivel medio superior.

En la primera etapa se consultó a quince practicantes (cinco mujeres y nueve hombres) expertos en AP, pertenecientes a una institución certificada para la enseñanza de la filosofía budista y la práctica de la AP, quienes evaluaron la suficiencia y relevancia (cf. Escobar y Cuervo, 2008), así como el estado y rasgo de los reactivos respecto a la definición de Brown y Ryan (2003) que integra la versión española del MAAS (Soler et al., 2012). Los criterios de inclusión de los participantes fueron el tener experiencia de al menos dos años de práctica con maestros acreditados en AP y estar clínicamente sanos. En la segunda etapa se solicitó a cuatro lingüistas de habla hispana especialistas en letras inglesas que evaluaran la claridad y coherencia de los reactivos en relación con la versión original en inglés. En la tercera etapa participaron once expertos (siete mujeres y cuatro hombres) practicantes de AP pertenecientes a un centro de enseñanza de la filosofía budista y la práctica de la AP, quienes evaluaron la relevancia y suficiencia de la versión del español para México. En la cuarta etapa, con la intención de obtener un índice de consistencia interna de la versión en español para México, participaron 267 estudiantes voluntarios de nivel medio superior (160 mujeres y 107 
hombres), residentes en una institución nacional de educación agraria con modalidad de internado ubicada en el Estado de México (México).

Se necesitó la aprobación de los padres puesto que los alumnos eran menores de edad, consentimiento informado que se solicitó a través una llamada telefónica. Adicionalmente, los participantes firmaron su asentimiento para el uso de sus datos en el estudio. Los participantes mayores de 18 años únicamente firmaron un formato de consentimiento, de acuerdo con las normas para menores de edad que participan en protocolos de investigación (Altamirano et al., 2010).

\section{Instrumentos}

Se empleó la versión española del MAAS de Soler et al. (2012), ya que incluye un procedimiento de traducción del inglés al español y de retraducción del español al inglés del original de Brown y Ryan (2003). El instrumento consta de quince reactivos que se evalúan mediante una escala Likert con recorrido de 1 ("casi siempre") a 6 ("casi nunca"), los cuales miden un estado de atención consciente a tareas cotidianas. Para la aplicación del instrumento no se requiere que los evaluados tengan experiencia en la práctica de la AP. La puntuación final es el promedio aritmético de las calificaciones asignadas a cada pregunta; así, los resultados altos indican un mayor estado de atención-reconocimiento.

\section{Procedimiento}

En la primera etapa, se envió una invitación por escrito a 19 expertos practicantes de AP pertenecientes a centros certificados para su enseñanza, de los cuales 15 aceptaron participar. A estos últimos se les informó la finalidad de la investigación y las instrucciones para evaluar cada reactivo con cuatro niveles de valoración: 1) no cumple el criterio, 2) bajo nivel, 3) moderado nivel y 4) alto nivel, todo ello con base en la definición de AP propuesta por Brown y Ryan (2003). Dichas evaluaciones se recabaron mediante un cuestionario diseñado por Escobar y Cuervo (2008), el cual permite obtener un porcentaje para cada reactivo en cada categoría a partir del total de las valoraciones de los jueces. Además, el porcentaje mínimo considerado para que un reactivo sea aceptable es de $80 \%$ ya que $100 \%$ equivale a 16 puntos, esto es, un alto nivel en las cuatro categorías.

Durante la segunda etapa se les solicitó a los expertos lingüistas que evaluaran la semántica de las preguntas que integran el MAAS en las categorías de claridad y coherencia con la definición, bajo los mismos cuatro niveles de valoración sobre los que trabajaron los expertos practicantes de AP para crear una versión adecuada para México. En la tercera etapa, once expertos en AP valoraron la relevancia y suficiencia de cada reactivo del instrumento, cuyo porcentaje de acuerdos de esta sección se indica en la Tabla 1.

Tabla 1. Datos de los expertos practicantes de AP $(n=26)$.

\begin{tabular}{|l|c|c|}
\hline & $\begin{array}{c}\text { Primera etapa } \\
\text { de evaluación } \\
\text { Versión española }\end{array}$ & $\begin{array}{c}\text { Segunda etapa } \\
\text { de evaluación } \\
\text { Versión mexicana }\end{array}$ \\
\hline Practicantes de AP (mujeres/hombres) & $\mathrm{N}=15(5 / 9)$ & $\mathrm{N}=11(7 / 4)$ \\
\hline Edad (años) & $\mathrm{M}=35.73$ (D.E. $=7.79)$ & $\mathrm{M}=41.27($ D.E. $=13.34)$ \\
\hline Experiencia en práctica de AP (años) & 3.90 (D.E. $=3.79)$ & $4.18($ D.E. $=3.76)$ \\
\hline
\end{tabular}

En la cuarta etapa, para obtener la validación de la nueva versión del instrumento para México, se invitó a 310 estudiantes a responder de manera voluntaria el cuestionario de quince reactivos, siendo los criterios de inclusión el que los participantes estuvieran clínicamente sanos y pertenecieran a una escuela de nivel medio superior con modalidad de internado. Como criterios de exclusión se consideraron el reporte de consumir medicamentos en las últimas dos semanas y sufrir alteraciones del sueño, así como no completar la evaluación. La muestra se integró al final con 267 alumnos que cumplieron con los citados criterios. 


\section{Análisis estadístico}

Se realizó un análisis de cada reactivo según su porcentaje de frecuencias y distribución de respuestas: 1) "casi siempre", 2) "muy frecuente", 3) "algo frecuente", 4) "poco frecuente", 5) "muy poco frecuente" y 6) "casi nunca". Se realizó una prueba t para muestras independientes en relación con edad, sexo y factores para descartar diferencias entre grupos; después, un análisis de componentes principales con un método de rotación varimax con Kaiser, usando para ello el programa estadístico SPSS, versión 23 (IBM Corporation, 2015), así como un análisis factorial confirmatorio para un modelo de un solo factor y para otro de dos factores, lo que se llevó a cabo mediante el programa AMOS, versión 21 (IBM Corporation, 2012).

\section{RESULTADOS}

En los análisis de distribución de frecuencias ninguna opción de respuesta fue seleccionada por más del 37\% de participantes en los reactivos 1 al 14 . En el reactivo 15, 68\% los participantes seleccionaron la opción 6 ("casi nunca"). Los datos sociodemográficos de los expertos practicantes de AP que evaluaron las versiones de la escala en español de España y México se muestran en la Tabla 1. Los 267 estudiantes que respondieron el instrumento, de los cuales fueron 160 mujeres y 107 hombres, con una edad promedio de 18.32 años y una desviación estándar de 1.79 años, no mostraron diferencias significativas entre los puntajes de hombres y mujeres $(t[1.204]=-2.772, d f=265$, $p=0.27$, IC 95\%), Sin embargo, hubo diferencias entre hombres y mujeres respecto a los puntajes del factor de reconocimiento, es decir, las mujeres puntuaron más alto en este factor $(t$ [4.228] $=$ -3.744, $d f=265, p=0.041)$. Además, no hubo diferencias entre intervalos de edad, puntaje y factores del MAAS (Tabla 2).

Los porcentajes de acuerdos totales obtenidos en la segunda etapa de evaluación hecha por los expertos practicantes de AP, así como las evaluaciones de los expertos lingüistas, variaron (Tabla 3). Un total de catorce reactivos cumplieron con el mínimo de $80 \%$ de acuerdos en todas las categorías. Pero el reactivo 6 ("Me olvido del nombre de una persona tan pronto me lo dicen por primera vez") no cumplió el mínimo de acuerdo entre los participantes de AP en la categoría de suficiencia, aunque se conservó para mantener un porcentaje superior a $80 \%$ en las categorías de relevancia, coherencia y claridad.

Tabla 2. Valores promedio ( \pm error estándar) de los puntajes de los factores Atención y Reconocimiento, comparando entre los intervalos de edad de los participantes.

\begin{tabular}{|c|c|r|c|c|}
\hline \multirow{2}{*}{ Factores } & $\begin{array}{c}\text { Intervalos } \\
\text { de edad }\end{array}$ & \multicolumn{1}{|c|}{ N } & M (D.E.) & \multirow{2}{*}{ F } \\
\hline \multirow{4}{*}{ Atención } & 15 a 16 & 47 & $4,5798(0.12)$ & \multirow{4}{*}{$0.790^{*}$} \\
\cline { 2 - 4 } & 17 a 18 & 84 & $4,3318(0.11)$ & \\
\cline { 2 - 4 } & 19 a 20 & 101 & $4,3700(0.09)$ & \\
\cline { 2 - 4 } & 21 & 35 & $4,4643(0.05)$ & \\
\hline \multirow{4}{*}{ Reconocimiento } & 15 a 16 & 47 & $4,2500(0.16)$ & \multirow{4}{*}{$1.475^{* *}$} \\
\cline { 2 - 4 } & 17 a 18 & 84 & $4,4167(0.10)$ \\
\cline { 2 - 4 } & 19 a 20 & 101 & $4,2005(0.10)$ & \\
\cline { 2 - 4 } & 21 & 35 & $4,5929(0.17)$ & \\
\hline
\end{tabular}

Nota: $* p<0.500 * * p<0.222$

La estimación del análisis factorial exploratorio indica que el instrumento se conforma por dos factores: atención, para los reactivos 1 a $8(\alpha=$
$0.82)$ y reconocimiento para los reactivos 9 a 12 $(\alpha=0.73)$. Se muestra el gráfico de sedimentación de dos factores (Figura 1). 
Tabla 3. Porcentaje de acuerdos de la evaluación de la versión en español para México de los practicantes expertos y expertos lingüistas.

\begin{tabular}{|l|c|c|c|c|}
\hline \multicolumn{1}{|c|}{ REACTIVOS } & \multicolumn{2}{c|}{$\begin{array}{c}\text { Practicantes expertos } \\
\text { en AP }\end{array}$} & \multicolumn{2}{c|}{ Expertos lingüistas } \\
\cline { 2 - 5 } & Suficiencia & Relevancia & Coherencia & Claridad \\
\hline 1. Puedo sentir una emoción y no darme cuenta hasta más tarde. & 84.85 & 100.00 & 100.00 & 93.94 \\
\hline $\begin{array}{l}\text { 2. Rompo o derramo cosas por descuido, por no poner atención } \\
\text { o por estar pensando en otra cosa. }\end{array}$ & 81.82 & 83.33 & 91.67 & 93.94 \\
\hline 3. Se me dificulta mantenerme enfocado en lo que ocurre en el presente. & 88.10 & 83.33 & 91.67 & 96.97 \\
\hline $\begin{array}{l}\text { 4. Suelo caminar rápidamente para llegar a donde voy, sin prestar } \\
\text { atención a lo que me pasa en el camino. }\end{array}$ & 93.94 & 91.67 & 100.00 & 90.91 \\
\hline $\begin{array}{l}\text { 5.Tiendo a no darme cuenta de alguna incomodidad o tensión física, } \\
\text { hasta que realmente llaman mi atención. }\end{array}$ & 87.88 & 83.33 & 91.67 & 93.94 \\
\hline $\begin{array}{l}\text { 6. Me olvido del nombre de una persona tan pronto me lo dicen } \\
\text { por primera vez. }\end{array}$ & $75.76 *$ & 83.33 & 91.67 & 81.82 \\
\hline $\begin{array}{l}\text { 7. Parece como si funcionara en “automático", sin darme cuenta } \\
\text { de lo que estoy haciendo. }\end{array}$ & 90.91 & 83.33 & 83.33 & 100.00 \\
\hline $\begin{array}{l}\text { 8. Realizo mis actividades rápidamente sin prestarles realmente } \\
\text { atención. }\end{array}$ & 81.82 & 83.33 & 100.00 & 93.94 \\
\hline $\begin{array}{l}\text { 9.Me concentro tanto en el objetivo que deseo alcanzar, que pierdo } \\
\text { contacto con lo que estoy haciendo en el presente. }\end{array}$ & 87.88 & 83.33 & 91.67 & 96.97 \\
\hline $\begin{array}{l}\text { 10. Hago trabajos o tareas automáticamente, sin darme cuenta de lo } \\
\text { que estoy haciendo. }\end{array}$ & 81.82 & 91.67 & 91.67 & 93.94 \\
\hline $\begin{array}{l}\text { 11. Me descubro a mí mismo escuchando a alguien sin prestar atención } \\
\text { y al mismo tiempo haciendo otra cosa. }\end{array}$ & 84.85 & 100.00 & 100.00 & 93.94 \\
\hline $\begin{array}{l}\text { 12. Me dirijo a lugares en "piloto automático" y luego me pregunto } \\
\text { por qué fui ahí. }\end{array}$ & 81.82 & 91.67 & 100.00 & 90.91 \\
\hline $\begin{array}{l}\text { 13. Me descubro pensando demasiado en el futuro o en el pasado. } \\
\text { 14. Noto que hago cosas sin prestar atención. }\end{array}$ & 90.91 & 83.33 & 100.00 & 100.00 \\
\hline 15. Pruebo alimentos sin darme cuenta de lo que estoy comiendo. & 84.85 & 83.33 & 100.00 & 100.00 \\
\hline
\end{tabular}

*Porcentajes que no cumplen el criterio $\geq 80 \%$ de acuerdo.

Figura 1. Gráfico de sedimentación del análisis de la versión en español para México con doce reactivos.

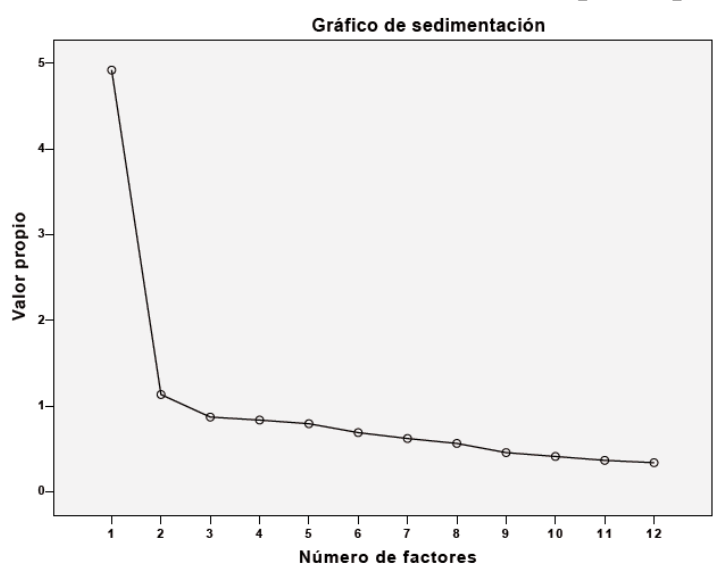

El coeficiente $\alpha$ de confiabilidad total del MAAS en su versión en español de México fue de 0.86 , con una varianza total explicada de $50.47 \%$ y un índice KMO de 0.89 obtenido mediante la prueba de esfericidad de Barlett ( $p<0.0001)$. Además, se observa que las cargas factoriales de cada reactivo fueron heterogéneas (Tabla 4). 
Tabla 4. Cargas factoriales de los componentes rotados para doce reactivos.

\begin{tabular}{|l|c|c|}
\hline \multicolumn{1}{|c|}{ REACTIVOS } & \multicolumn{2}{c|}{ SUBESCALAS } \\
\cline { 2 - 3 } & Atención & Reconocimiento \\
\hline $\begin{array}{l}\text { 4. Tiendo a no darme cuenta de alguna incomodidad o tensión física hasta que realmente } \\
\text { llaman mi atención. }\end{array}$ & 0.75 & \\
\hline 6. Realizo mis actividades rápidamente sin prestarles atención. & 0.72 & \\
\hline 8.Hago trabajos o tareas automáticamente sin darme cuenta de lo que estoy haciendo. & 0.65 & \\
\hline 5. Parece como si funcionara en "automático" sin darme cuenta de lo que estoy haciendo. & 0.63 & \\
\hline $\begin{array}{l}\text { 3. Suelo caminar rápidamente para llegar a donde voy, sin prestar atención a lo que me pasa } \\
\text { durante el camino. }\end{array}$ & 0.63 & \\
\hline $\begin{array}{l}\text { 7. Me concentro tanto en el objetivo que deseo alcanzar, que pierdo contacto con lo que estoy } \\
\text { haciendo en el presente. }\end{array}$ & 0.59 & \\
\hline 2. Se me dificulta mantenerme enfocado en lo que ocurre en el presente. & 0.38 \\
\hline $\begin{array}{l}\text { 1. Rompo o derramo cosas por descuido, por no poner atención, o por estar pensando } \\
\text { en otra cosa. }\end{array}$ & 0.48 & \\
\hline 12. Noto que hago cosas sin prestar atención. & & \\
\hline 11. Me descubro pensando demasiado en el futuro o en el pasado. & & \\
\hline 10. Me dirijo a lugares en "piloto automático" y luego me pregunto por qué fui ahí. & & \\
\hline $\begin{array}{l}\text { 9. Me descubro a mí mismo escuchando a alguien sin prestar atención y al mismo tiempo } \\
\text { haciendo otra cosa. }\end{array}$ & & 0.74 \\
\hline Correlaciones intersubescalas =.60* & & 0.71 \\
\hline Número de reactivos & 8 & \\
\hline Varianza por cada componente & $29.31 \%$ & $21.16 \%$ \\
\hline M (D.E.) & $35.25(7.57)$ & $17.31(4.28)$ \\
\hline Media teórica & $4.40(0.94)$ & $4.32(1.07)$ \\
\hline "Cocficie & & \\
\hline
\end{tabular}

*Coeficiente de correlación entre factores, $p<0.001$

Por otra lado, la pregunta 7 ("Parece como si funcionara en automático sin darme cuenta de lo que estoy haciendo") perteneció a ambos factores, pero el mayor puntaje se mantuvo en el factor de atención, con una diferencia de 0.21 unidades. La escala final se conformó por doce reactivos, ya que se eliminaron las siguientes preguntas: 1 ( "Puedo sentir una emoción y no darme cuenta hasta más tarde"), 6 ("Me olvido del nombre de una persona tan pronto me lo dicen por primera vez") y 15 ("Pruebo alimentos sin darme cuenta de qué estoy comiendo") debido a que estas preguntas no aportaron significativamente al índice de consistencia interna y mostraron una carga factorial in- suficiente respecto a la escala $(0.29,0.20$ y 0.30 , respectivamente), y por tanto se omitieron en el análisis factorial.

\section{Análisis factorial confirmatorio}

Los índices de ajuste para un modelo de dos factores es superior al modelo de un factor (Tabla 5) y se encuentran dentro de los intervalos aceptables que marcan Hu y Bentler (1999): $\chi 2<3$; CFI > .90; GFI $>.95 ;$ AGFI $>.80 ;$ RMR $<.09 ;$ RMSEA $<.05$; PCLOSE $>.05$ (Tabla 5).

Así, se obtuvo el siguiente modelo factorial para la escala del MAAS (Figura 2).

Tabla 5. Índices de ajuste del modelo de dos factores comparado con el modelo de un factor.

\begin{tabular}{|c|c|c|c|c|c|c|c|}
\hline Factor & Chi2 & CFI & GFI & AGFI & RMR & RMSEA & PCLOSE \\
\hline 1 & 2.63 & .914 & .921 & .884 & .110 & .078 & .002 \\
\hline 2 & 2.02 & .946 & .940 & .911 & .091 & .062 & .119 \\
\hline
\end{tabular}


Figura 2. Modelo bifactorial (Atención y Reconocimiento) del Mindful Attention Awarness Scale (MAAS) para estudiantes de nivel medio superior.

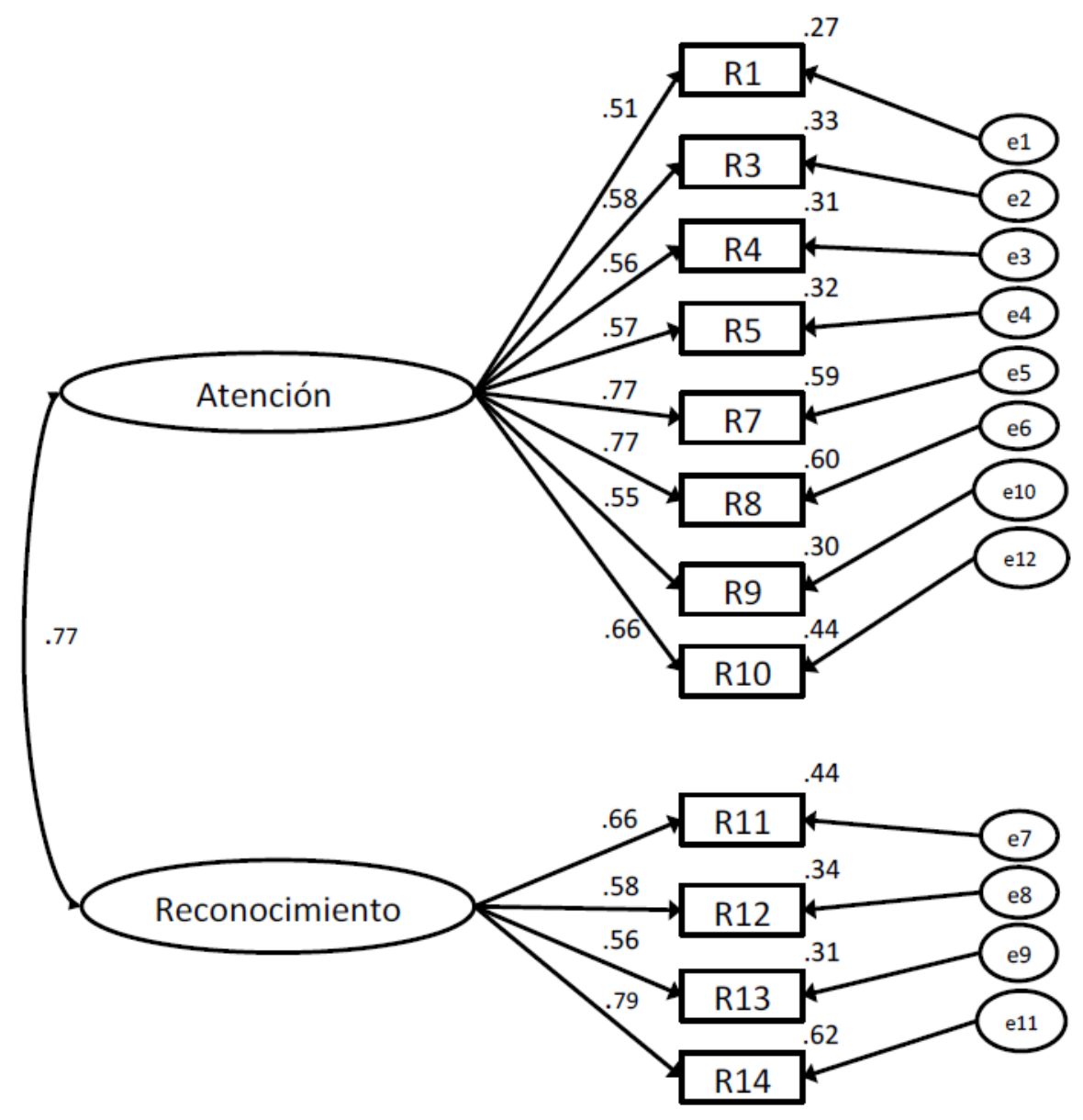

\section{DISCUSIÓN}

La principal contribución del estudio consiste en mostrar las diferencias de los resultados reportados de las adaptaciones del MAAS por otros autores, tanto en España (Inchausti et al., 2014; Soler et al., 2012), Alemania (Bruin et al., 2011) como en México (López-Maya et al., 2015), en las cuales se ha sugerido un solo factor. En el presente estudio se demostró que hay dos factores, con una varianza de $50.47 \%$ e índices de ajuste superiores a un modelo de un solo factor, mientras que López-Maya et al. (2015) reportaron 41\% de la varianza, similar a la de Soler et al. (2012). Cabe señalar que los índices RMR y RMSEA en este estudio están cerca del límite inferior establecido, y no son suficientes, como ocurre en otras investigaciones. Por ejemplo, Bruin et al. (2011) reportan un RMSEA de .035. Además, entre las principales aportaciones de la presente investigación se en- cuentran el haber incorporado la variable referente a la maduración cognitiva en los jóvenes, así como haber sometido a prueba la utilidad del factor de reconocimiento en los programas basados en la AP y la diferencia de puntajes entre hombres y mujeres en el factor de reconocimiento.

Los reactivos 9 ("Me descubro a mí mismo escuchando a alguien sin prestar atención y al mismo tiempo haciendo otra cosa"), 10 ("Me dirijo a lugares en "piloto automático y luego me pregunto por qué fui ahí"), 11 ("Me descubro pensando demasiado en el futuro o en el pasado") y 12 ("Noto que hago cosas sin prestar atención") se agruparon en el segundo factor, al cual se denominó "reconocimiento" debido a que contienen palabras que se relacionan con el "darse cuenta", es decir, "noto" "descubro" "me pregunto" son frases que difieren del primer factor ("atención”), que contiene oraciones relacionadas con actividades, tareas y sensaciones. La contribución de los 
dos factores en los estudiantes de nivel medio superior podría deberse a que en ellos la percepción, la atención y la conciencia usan vías distintas que aún no se han integrado como un único proceso, según como lo sugieren Brown y Ryan (2003), razón por la cual los participantes identifican la atención y el reconocimiento por separado.

El análisis de los reactivos muestra que el reactivo 1 ("Puedo sentir una emoción y no darme cuenta hasta más tarde”) aportó una carga factorial inferior a 0.30 , por lo que se descartó su uso en la prueba en cuanto que dicho elemento tendía hacia calificaciones del tipo "nunca" en la escala Likert, produciendo así una nula variabilidad de respuestas. El reactivo 6 ("Me olvido del nombre de una persona tan pronto me lo dicen por primera vez") fue uno de los más inciertos, ya que su estructura no aportó a ningún factor en el análisis factorial exploratorio; asimismo, fue el único reactivo que no cumplió con el $80 \%$ de acuerdos entre jueces expertos en las categorías de suficiencia y relevancia. Es posible que los participantes pudieran comprender la frase "me olvido" como una pregunta relacionada a la memoria más que a la falta de "atención; por ejemplo Grossman (2011) sugiere que la escala MAAS es inapropiada porque utiliza preguntas relacionadas con la carencia de atención más que a la atención en sí; sin embargo, los reactivos tienen la finalidad de hacer consciente la experiencia que se describe en cada enunciado, por lo que el esforzarse en reconocer la falta de atención de quien responde el instrumento invita a poner mayor énfasis en los estímulos tanto externos como internos, pues el no estar atento es más fácil de reconocer por las personas sin entrenamiento en la AP (Brown, West, Loverich y Biegel, 2011). Finalmente, el reactivo 15 ("Pruebo alimentos sin darme cuenta de qué estoy comiendo”) no aportó la suficiente carga factorial al instrumento; además, los participantes puntuaron en su mayoría "nunca" en la escala Likert.

Es importante destacar que Inchausti et al. (2014) eliminaron las preguntas 9, 12 y 5 de la versión española; en el caso del reactivo 9, estos autores refieren un desajuste en sus pruebas de pretest y postest y una redacción abstracta y redundante. Por otro lado, los expertos lingüistas modificaron el reactivo para la prueba en el es- pañol de México: "Me concentro tanto en el objetivo que deseo alcanzar, que pierdo contacto con lo que estoy haciendo en el presente". Después de dicha modificación, este reactivo mostró una carga factorial de 0.59 y no presentó dificultades. En el caso del reactivo 12, eliminado también por los autores en su versión española, refieren que no mostró un buen ajuste y consideran que la pregunta es redundante respecto a la 7 , en la que se emplea la frase "piloto automático". Por ende, los expertos lingüistas que participaron en sta investigación sugirieron modificar la pregunta, redactándola como "Me dirijo a lugares en piloto automático, y luego me pregunto por qué fui ahi'" sustituyendo así la palabra "conducir" ya que en México dicha palabra se asocia a manejar un automóvil. Tras dicha modificación el reactivo arrojó una carga factorial de las más altas: 0.71 , pero se ubicó como un segundo factor de reconocimiento. Esas modificaciones coinciden con la observación de Inchausti et al. (2014), quienes sugieren modificar el reactivo utilizando la frase "voy a lugares," en vez de "conduzco". Dichos autores también prescindieron del reactivo puesto que mostraba ambigüedad semántica, pues en español no es posible darse cuenta de algo sin que capte la atención, y la pregunta no hace énfasis en la intensidad de ese "darse cuenta"; es decir, la mínima suficiente para que capte nuestra atención. Por tanto, en nuestra versión para México se modificó la pregunta como "Tiendo a no darme cuenta de alguna incomodidad o tensión física hasta que realmente llaman mi atención", en que se elimina la palabra "sensación" por considerarse una emoción, y sustituyendo "captar" por "llamar", palabra que indica que debe haber un estímulo lo suficientemente intenso para que sea posible percatarse de él; en este sentido, debe considerarse la importancia del uso e interpretación de las palabras cada vez que se traduce, adapta o se crea una escala psicométrica.

En escenarios clínicos se enseña a los pacientes a diferenciar entre "atender" a los pensamientos y "reconocer" que los experimentan. Un ejemplo de este proceso es la llamada "defusión cognitiva", que se define como "observar los pensamientos para analizarlos sin juicio" y considerarlos como lo que son: solamente pensamientos (Pérez, 2012). Por tanto, el considerar la atención 
y el reconocimiento como dos procesos básicos es consistente con el entrenamiento en AP en cuanto a atender y "dejar ir" los pensamientos, reconociéndolos como productos que se generan en la mente. A su vez, Kang, Gruber y Gray (2013) refieren que, al parecer, son necesarias cuatro habilidades como mínimo y no solo un factor de atención-reconocimiento para producir la experiencia de AP: ser conscientes de los eventos internos o externos; tener una atención sostenida, alejando de la conciencia las posibles distracciones; focalizarse en el momento presente, y aceptar sin criticar cualquier cosa que suceda, de los cuales los tres primeros procesos se refieren a atender el evento y el último a la aceptación.

Es posible sugerir, al igual que otros autores, que el entrenamiento en AP contribuye al desarrollo de la metacognición, la cual se define como la capacidad de monitorear y controlar los procesos cognitivos propios (Shimamura, 2000). Además, dicho fenómeno no se considera una función ejecutiva, sino el proceso de mayor jerarquía cognitiva por su relación con la corteza prefrontal (CPF) (Fernandez, Baird y Posner, 2000).

Algunas investigaciones sugieren que hay cambios en la CPF después de un entrenamiento en AP. Por ejemplo, Farb et al. (2007) reportaron un incremento de la actividad en la CPF después de ocho semanas de haber implementado el programa de AP para reducir el estrés en participantes sanos, independientemente de su edad y sexo. De igual forma, en esta investigación no se obtuvieron diferencias entre los intervalos de edad, resultado consistente con el proceso metacognitivo, es decir, con el monitoreo y el control que se realiza en los procesos cognitivos más básicos, los cuales se van adquiriendo paulatinamente mediante las técnicas de meditación que emplean los programas de AP, así como dirigiendo la atención a la respiración. Un ejemplo de proceso metacognitivo más elaborado involucra la comparación entre el presente y el estado en que desearía estar (Schooler, 2002). Durante este proceso de autoevaluación se compara y se reconoce la discrepancia entre el estado actual ("Me estoy reprochando y me siento ansioso"), el estado autoevaluado ("Reconozco que un estado de ansiedad me produce rumiar") y el estado deseado ("Dejaré de rumiar, lo que me hará sentir diferente"). Dicho proceso de autoevaluación metacognitiva permite la regulación y el decremento de la preocupación, la ansiedad y el estrés (Holas y Jankowski, 2013), así como el aumento de una actitud de autocuidado (Hollis-Walker y Colosimo, 2011) y un mejor rendimiento académico (Bluth et al., 2015; Huppert y Johnson, 2010; Kuyken et al., 2013). Tanto el proceso de atención y reconocimiento como la autoevaluación entre el estado actual y el deseado son procesos que dirigen la atención cuando esta falta. Por lo tanto, la falta de atención es tan importante como la atención misma, pues permite el desarrollo de la metacognición mediante el atender, reconocer y corregir.

Las limitaciones de este estudio fueron el no haber tenido una muestra clínica como grupo de contraste. Además, se considera sumamente importante incluir en futuras evaluaciones algunas variables neuropsicológicas que aporten un mayor conocimiento del efecto de maduración metacognitiva acelerada debido al entrenamiento en AP.

A pesar de que no hubo diferencias en cuanto al efecto de la edad de los participantes en los puntajes del MAAS -lo que es consistente con los resultados de Brown y Ryan (2003) en cuanto a que los programas de entrenamiento en AP mejoran la atención independientemente de la edad-, se desconocen los cambios en el factor de reconocimiento, por lo que se sugiere incluir evaluaciones de la atención sostenida, puesto que el diseño del MAAS, como un autorreporte, no permite conocer con precisión la capacidad de los evaluados al referir su propio nivel de atención y reconocimiento.

Es importante continuar evaluando la AP en grupos diversos de la población mexicana con la intención de ahondar en su conocimiento y poder así hacer propuestas terapéuticas específicas en el campo de la psicología de la salud en el país, que recién inicia la instrumentación clínica controlada de los tratamientos basados en la AP. 


\section{REFERENCIAS}

Altamirano B., N., Altamirano B., E., Olaya V., A., De Rubens, J., García P., S. y Altamirano B., M.M. (2010). Consentimiento informado en grupos vulnerables: participación de niños y adolescentes en protocolos de investigación. Boletín de Medicina del Hospital Infantil de México, 67, mayo-junio, 248-258.

Bluth, K., Campo, R.A., Pruteanu-Malinici, S., Reams, A., Mullarkey, M. y Broderick, P.C. (2015). School-based mindfulness pilot study for ethnically diverse at-risk adolescents, Mindfulness, 7(1), 90-104. doi: 10.1007/ s12671-014-0376-1.

Brown, K.W. y Ryan, R.M. (2003). The benefits of being present: Mindfulness and its role in psychological well-being. Journal of Personal and Social Psychology, 84, 822-848. doi: 10.1037/0022-3514.84.4.822.

Brown, K.W., West, A.M., Loverich, T.M. y Biegel, G.M. (2011). Assessing adolescent mindfulness: validation of an adapted Mindful Attention Awareness Scale in adolescent normative and psychiatric populations. Psychological Assessment, 23(4), 1023-1033. doi: 10.1037/a0021338.

Bruin, E.I., Zijlstra, B.J. y Veirjer-Bergsma, S.M.B. (2011). The Mindful Attention Awareness Scale for Adolescents (MAAS-A): psychometric properties in a Dutch sample. Mindfulness, 2, 201-211. doi: 10.1007/s12671-011-0061-6.

Campanella, F., Crescentini, C., Urgesi, C. y Fabbro, F. (2014). Mindfulness-oriented meditation improves self-related character scales in healthy individuals. Comprehensive Psychiatry, 55, 1269-1278. doi: 10.1016/j.comppsych.2014.03.009.

Chambers, R., Gullone, E. y Allen, N.B. (2009). Mindful emotion regulation: An integrative review. Clinical Psychology Review, 29, 560-572.

Escobar P., J. y Cuervo M., A. (2008). Validez de contenido y juicio de expertos: una aproximación a su utilización. Avances en Medición, 6, 27-37.

Farb, N.S., Segal, Z.V., Mayberg, H., Bean, J., McKeon, D., Fatima, Z., et al. (2007). Attending to the present: Mindfulness meditation reveals distinct neural modes of self reference. Social Cognitive and Affective Neuroscience, 2(4), 313-322. doi: $10.1093 / \mathrm{scan} / \mathrm{nsm} 030$.

Fernandez D., D., Baird, J.A. y Posner, M.I. (2000). Executive attention and metacognitive regulation. Consciousness and Cognition, 9(2), 288-307. doi: 10.1006/ccog.2000.0447.

Gregoski, M.J., Barnes, V.A., Tingen, M.S., Harshfield, G.A. y Treiber, F.A. (2011). Breathing awareness meditation and life skills training programs influence upon ambulatory blood pressure and sodium excretion among African American adolescents. Journal of Adolescent Health, 48, 59-4. doi: 10.1016/j.jadohealth.2010.05.019.

Grossman, P. (2011). Defining mindfulness by how poorly I think I pay attention during everyday awareness and other intractable problems for psychology's (re)invention of mindfulness. Comment on Brown et al. (2011). Psychological Association, 23(4), 1034-1040. doi: 10.1037/a0022713.

Holas, P. y Jankowski, T. (2013). A cognitive perspective on mindfulness. International Journal of Psychology, 48(3), $232-243$. doi: 10.1080/00207594.2012.658056.

Hollis-Walker, L. y Colosimo, K. (2011). Mindfulness, self-compassion, and happiness in non-meditators: A theoretical and empirical examination. Personality and Individual Differences, 50(2), 222-227. doi: 10.1016/j.paid.2010.09.033.

Hu, L. y Bentler, P.M. (1999). Cutoff criteria for fit indexes in covariance structure analysis: Conventional criteria versus new alternatives. Structural Equation Modeling: A Multidisciplinary Journal, 6(1), 1-55. doi: 10.1080/10705519909540118.

Huppert, F.A. y Johnson, D.M. (2010). A controlled trial of mindfulness training in schools: the importance of practice for an impacton well-being. Journal of Positive Psychology, 5(4), 264-274. doi: 10.1080/17439761003794148.

IBM Corporation (2012). SPSS Statistics for Windows, Version 21.0. Armonk, NY: IBM Corp.

IBM Corporation (2015). SPSS Statistics for Macintosh, Version 23.0. Armonk, NY: IBM Corp.

Inchausti, F., Prieto, G. y Delgado, A.R. (2014). Analisis rasch de la versión española de la Mindful Attention Awarness Scale en una muestra clínica. Revista de Psiquiatría y Salud Mental, 7(1), 32-41. doi: 10.1016/j.rpsmen.2013.07.001.

Kabat-Zinn, J., Lipworth, L. y Burney, R. (1985) The clinical use of mindful-ness meditation for the self-regulation of chronic pain. Journal of Behavioral Medicine, 8, 163-90. doi: 10.1007/BF00845519.

Kang, Y., Gruber, J. y Gray, J.R. (2013). Mindfulness and de-automatization. Emotion Review, 5(2), 192-210. doi: doi.org/ $10.1177 / 1754073912451629$.

Keng, S-L., Smoski, M.J. y Robins, C.J. (2011). Effects o mindfulness on psychological health: A review of empirical studies. Clinical Psychological Review, 31, 1041-1056. doi: 10.1016/j.cpr.2011.04.006.

Khoury, B., Sharma, M., Rush, S.E. y Fournier, C. (2015). Mindfulness-based stress reduction for healthy individuals. Journal of Psychosomatic Research, 78, 519-528. doi: 10.1016/j.jpsychores.2015.03.009.

Kuyken, W., Weare, K., Ukoumunne, O.C., Vicary, R., Motton, N., Burnett, R., Cullen, C., Hennelly, S. y Huppert, F. (2013). Effectiveness of the mindfulness in schools programme: non-randomised controlled feasibility study. British Journal of Psychiatry, 203(2), 126-131. doi: 10.1192/bjp.bp.113.126649. 
López-Maya, E., Hernández P., M.R., Hölzel, B.K., Méndez S., L., Gutiérrez G., J.J., Araujo D., D., Nuñez G., A., Cervantes S., L.K., González F., L.E., Nava A., S. y Bautista G., L.E. (2015). Psychometrics properties of the Mexican version of the Mindfulness Attention and Awareness Scale. Psychologia. Avances de la Disciplina, 9(1), 13-27.

Meda-Lara, R.M., Herrero, M., Blanco D., L.M., Moreno J., B. y Palomera C., A. (2015). Propiedades psicométricas del Cuestionario de Cinco Facetas de la Conciencia Plena. (Five Facet Mindfulness Questionnaire, FFMQ-M) en México. Psicología Conductual, 23(3), 467-487.

Pérez Á., M. (2012). Third-generation therapies: Achievements and challenges. International Journal of Clinical and Health Psychology, 12(2), 291-310.

Rapgay, L. y Bystrisky, A. (2009). Classical mindfulness: An introduction to its theory and practice for clinical application. Annual New York Academic of Sciences, 1172, 148-162. doi: 10.1111/j.1749-6632.2009.04405.x.

Santiago M., J. (2016). Intervención basada en atención plena sobre depresión y atracón en pacientes candidatos a cirugía bariátrica. Tesis inédita de maestría. Ciudad de México: Universidad Nacional Autónoma de México.

Schooler, J.W. (2002). Re-representing consciousness: Dissociations between experience and meta-consciousness. Trends in Cognitive Sciences, 6(8), 339-344.

Shimamura, A.P. (2000). Toward a cognitive neuroscience of metacognition. Consciousness and Cognition, 9, 313-323.

Soler, J., Tejedor, R., Feliu-Soler, A., Pascual, J.C., Cebolla, A., Soriano, J., et al. (2012). Propiedades psicométricas de la versión española de la Mindful Attention Awareness Scale (MAAS). Acta Española de Psiquiatria, 40(19), 26-37.

Teasdale, J.D., Segal, Z. y Williams, J.M., (1995). How does cognitive therapy prevent depressive relapse and why should attentional control (mindfulness) training help. Behavioral Research Therapy, 33, 25-39. 